\section{A Case Report of Hemodialysis Graft-induced Increased Intraocular Pressure}

\section{Dear Editor,}

Hemodialysis is performed in the patients with end-stage renal disease, and arteriovenous fistula (AVF) is regarded as the best type of hemodialysis access [1]. However, various complications, such as thrombosis, stenosis and infection have been reported [2]. In the field of ophthalmology, rare cases of increased intraocular pressure (IIOP) related to hemodialytic condition have been reported [2]. In this study, we report the case of a patient who presented with IIOP, severe headache, and prolonged choroidal detachment due to abnormal venous drainage.

A 50-year-old Korean man, with end-stage renal disease and in a hemodialysis program for 8 years, presented with IIOP in his left eye. His right eye was blinded due to a history of surgery for retinal detachment, and he was undergoing treatment for chronic open-angle glaucoma in his left eye. Visual acuity was $20 / 25$, IOP was $18 \mathrm{mmHg}$, and spherical equivalent was -0.375 diopter. Ocular examination revealed regular pseudophakia, axial length of $23.13 \mathrm{~mm}$ and central corneal thickness of $507 \mu \mathrm{m}$. Fundus exam showed a pale optic disc and a diffuse superior thinning of the retinal nerve fiber layer. He underwent trabeculectomy due to IIOP even with the maximum tolerated medical therapy. After surgery, the IOP decreased to 9 $\mathrm{mmHg}$, but choroidal detachment was developed. Nine days after surgery, he presented with severe headache and ocular pain. The IOP increased to $25 \mathrm{mmHg}$, and functional filtration bleb formed by trabeculectomy was not well elevated. In addition, although the IOP was elevated, no significant improvement in choroidal detachment was observed (Fig. 1A, 1B). Afterwards, the status of choroidal detachment tended to wax and wane, although the IOP was

Received: March 19, 2021 Final revision: May 9, 2021

Accepted: May 14, 2021
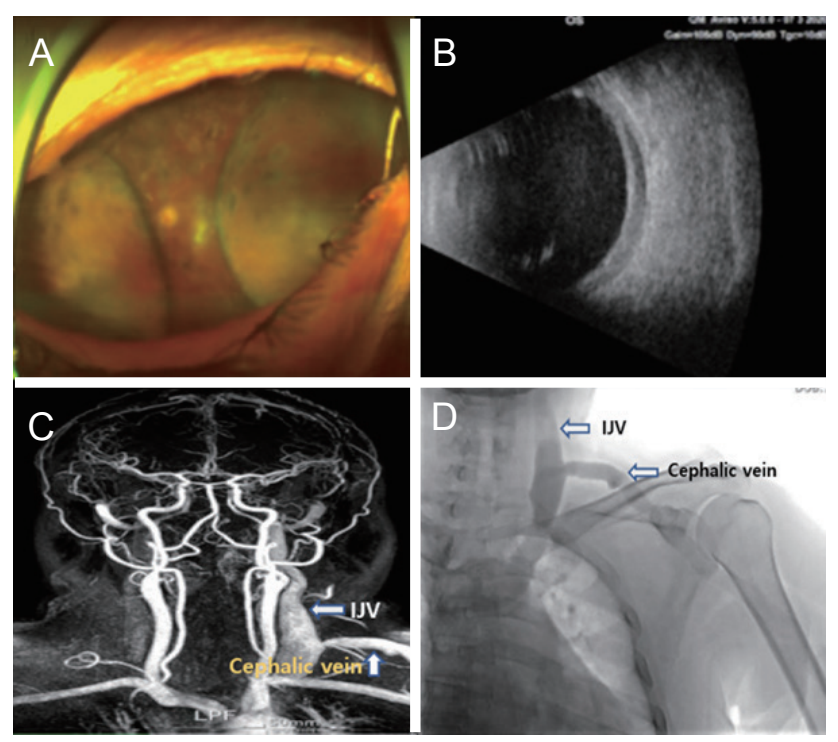

Fig. 1. Photographs of choroidal detachments 9 days after trabeculectomy on (A) color ultra-widefield fundus photography and (B) B-scan ultrasonography. The condition was not improved even though the postoperative hypotony resolved. (C) Magnetic resonance angiography shows flow signals of left internal jugular vein (IJV) and superior sagittal sinus and cavernous sinus in the arterial phase, suggesting venous reflux. (D) Fistulography shows invisible cephalo-axillary junction or cephalic arch, revealing the cephalic vein crosses the left clavicle and joins the IJV instead of the subclavian vein.

maintained relatively high.

He underwent brain work-up, and result showed flow signs on left internal jugular vein (IJV) with a significant dilatation in the arterial phase (Fig. 1C). Due to the suspicion that the IIOP may be related to an abnormality in the hemodialytic vessels, he underwent fistulography to check the arteriovenous flow. Surprisingly, fistulography confirmed that the venous flow of the cephalic vein in the AVF circuit was directly joined to the IJV, and not to the subclavian vein, inducing venous reflux to the brain (Fig. 1D). He underwent a left cephalic vein ligation and new AVF formation in the right side. After surgery, the IOP decreased to $16 \mathrm{mmHg}$ on the first day and $6 \mathrm{mmHg}$ on the second day, and the patient's subjective symptoms also improved. After two weeks, IOP was stable and the visual acuity was improved to 20 / 40. In addition, a remarkable improvement of choroidal detachment over time was observed, which completely resolved after two months. He was dis- 
charged and, at regular visits, is being monitored for disease progress.

There have been a few reports of IIOP in the patients on hemodialysis due to the abnormal venous flow into the external jugular vein (EJV) [2,3]. Jun et al. [3] reported abnormal venous drainage to the EJV in the patients on hemodialysis. Gomes et al. [2] also argued about the importance of confirming the IIOP when symptoms such as headache occur in the patients on hemodialysis. However, our report is more significant because of the venous drainage to the IJV, as most previous studies have reported venous drainage to the EJV $[2,4]$. Normal venous flow of graft vessels begins in radio-cephalic anastomosis of AVF, and the cephalic vein passes through the cephalo-axillary junction and the subclavian vein, entering the right atrium of heart [5]. The venous valves of the jugular vein act to prevent abnormal venous reflux. There is one competent venous valve at the level of junction with the subclavian vein in the IJV. In this case, a malfunction of venous valve due to a high flow in the junction of IJV could explain a sudden onset of symptoms. In addition, while the mechanism of repeated improvement and deterioration of choroidal detachment remains poorly understood, it is thought to fluctuate according to the dural sinus pressure.

This report focuses on a rare case of neuro-ophthalmologic manifestation caused by subclavian-jugular reflux in a patient on hemodialysis. It reminds us of the importance of discriminating the likelihood of such secondary glaucoma formation in the patients on hemodialysis when accompanied by neuro-ophthalmologic signs and symptoms.
Kang Min Lee, Choul Yong Park, Martha Kim

Department of Ophthalmology, Dongguk University Ilsan Hospital, Goyang, Korea

E-mail (Martha Kim): marthakim22@med.dongguk.ac.kr

\section{Conflict of Interest}

No potential conflict of interest relevant to this article was reported.

\section{References}

1. Choinski KN, Sundick SA, Rao AG, et al. The current role of the percutaneous arteriovenous fistula for hemodialysis access. Surg Technol Int 2020;37:217-24.

2. Gomes CM, Lucon AM, Yamada RT, et al. Rare complication of an arteriovenous fistula for dialysis: glaucoma. Scand J Urol Nephrol 2000;34:219-21.

3. Jun ESW, Lun ALY, Nikam M. A rare anatomic variant of a single-conduit supraclavicular cephalic arch draining into the external jugular vein presenting with recurrent arteriovenous fistula stenosis in a hemodialysis patient. $J$ Vasc Surg Cases Innov Tech 2017;3:20-2.

4. Ho YJ, Yeh CH, Lai CC, et al. ExPRESS miniature glaucoma shunt for intractable secondary glaucoma in superior vena cava syndrome: a case report. BMC Ophthalmol 2016;16:125.

5. Martin C 3rd, Pillai R. Dialysis access anatomy and interventions: a primer. Semin Intervent Radiol 2016;33:52-5. 